\title{
Ideen und Diskurse im Kontext der ökonomischen Bewertung von Ökosystemleistungen
}

\author{
Spätestens seit dem von den Vereinten Nationen \\ ins Leben gerufenen Millennium Ecosystem \\ Assessment steht das Konzept der Ökosystem- \\ leistungen hoch auf der politischen Agenda. \\ Ökosystemleistungen sind der aktuellste Versuch, \\ den Wert der Natur verstärkt in die politische \\ Entscheidungsfindung einzubinden. Welche Rolle \\ spielen dabei Ideen und Diskurse? \\ Von Alexandra Dehnhardt und Ulrich Petschow
}

D em Millennium Ecosystem Assessment (MA 2005) zufolge werden als Ökosystemleistungen (ÖSL) die Nutzen, die Ökosysteme für den Menschen erbringen, bezeichnet. Mithin wird hier eine anthropozentrische Perspektive eingenommen und der Wert der Leistungen für das menschliche Wohlergehen in den Vordergrund gestellt (Naturkapital Deutschland TEEB DE 2012). Die Brückenfunktion zwischen einer naturund sozialwissenschaftlichen Perspektive wurde nun expliziter. Mit der Erfassung und Darstellung des (ökonomischen) Wertes soll die Bedeutung von Natur und ÖSL für die Gesellschaft hervorgehoben werden, um so ein zusätzliches Argument für den Erhalt und ein nachhaltiges Management von Ökosystemen zu liefern und verstärkt Rationalität und Transparenz in den naturschutzpolitischen Entscheidungsprozess zu bringen.

Die Operationalisierung des Konzeptes und dessen Implementierung in die praktische Politik gestalten sich jedoch sehr schwierig. Dies liegt einerseits daran, dass es offensichtlich einen institutionellen mis-fit zwischen dem ÖSL-Konzept und den prozeduralen und organisationalen Anforderungen der Entscheidungsfindung gibt, der noch nicht hinreichend gelöst ist (Laurans/Mermet 2014). Neue Ideen und Konzepte werden von bestehenden Institutionen unter bestimmten Bedingungen aufgenommen und zugleich interpretiert, um diese für den bestehenden Kontext bearbeitbar zu machen. So wurde am Beispiel der Wasserwirtschaft gezeigt, dass die von der europäischen Wasserrahmenrichtlinie (WRRL) geforderte Integration ökonomischer Ansätze in (etablierte) politisch-administrative Entscheidungsprozesse diese unter einen erheblichen Anpassungsdruck stellt (Dehnhardt 2012; Martin-Ortega 2012) und die bestehenden nationalen Institutionen auf verschiede- nen Ebenen herausfordert: die handelnden Akteure und ihre Ressourcen, die Organisation und die Prozeduren sowie die Werte und gesellschaftlichen Ziele (Dehnhardt 2013). Andererseits ist jedoch auch festzustellen, dass das ÖSL-Konzept in unterschiedlichen wissenschaftlichen Disziplinen und politiknahen Netzwerken unterschiedlich interpretiert und rezipiert wird. $\mathrm{Ob}$ das Konzept und vor allem auch der ökonomische Wert von Natur als neue Idee Einfluss auf die Politikgestaltung nehmen und einen institutionellen Wandel bewirken kann, hängt ganz wesentlich von den vorherrschenden Problemwahrnehmungen der beteiligten Akteurskoalitionen, vom diskursiven Gehalt der Ideen und von der Stabilität des vorhandenen institutionellen Systems ab. Welchen Stellenwert neben den genannten institutionellen Herausforderungen vor allem auch differierende Ideen und Interpretationen des ÖSL Konzeptes für dessen Implementierung in die politische Praxis haben, ist bislang weder empirisch noch konzeptionell hinreichend untersucht.

Die Orientierung auf ÖSL und den ökonomischen Wert von Natur und Ökosystemen spiegelt eine diskursive Wendung in der Umwelt- und Naturschutzpolitik wider. Im folgenden Beitrag werden die Dynamiken dieses Diskurses betrachtet, insbesondere wird ein erster analytischer Blick auf die ideenbasierte Argumentation unterschiedlicher akademischer Diskurse und ihr Einfluss auf die Politikgestaltung geworfen. Dabei wird die Frage beleuchtet, wie Ideen und Diskurse die Gestaltung und das Ergebnis politischer Prozesse beeinflussen, und versucht, anhand der Verbreitung des ÖSL-Konzeptes die wesentlichen Dimensionen der Beeinflussung zu konzeptualisieren. Aus dieser Perspektive lassen sich erste Rückschlüsse ziehen, ob und wie das ÖSL-Konzept und das damit generierte neue Wissen vor allem aus ökonomischem Blickwinkel tatsächlich Eingang in die praktische Politikgestaltung finden kann.

\section{Die Bedeutung von Ideen für die Politikgestaltung}

In den letzten Jahrzehnten ist ein Bedeutungszuwachs ideenbezogener Ansätze in der Policy-Forschung zu verzeichnen (Campbell 2002; Béland et al. 2015). Diese stellen nicht den institutionellen Rahmen in den Mittelpunkt, sondern fokussieren vor allem auf die Rolle von Argumenten, Ideen und Überzeugungen zur Erklärung politischer Prozesse und politischen 
Wandels und ihren Einfluss auf Institutionen. So betonen Béland und Waddan (2011), dass - trotz der Gestaltungskraft politischer Institutionen - sowohl ein abrupter, aber auch inkrementelle Formen des politischen Wandels nicht ohne die Berücksichtigung der Rolle von Ideen erklärt und verstanden werden können. In diesem Zusammenhang hat sich als Ergänzung der bestehenden Formen des Neo-Institutionalismus, der die Rolle von formellen und informellen Institutionen als Determinanten der Handlungsmöglichkeiten einer Gesellschaft und damit als Bedingungen für Entwicklungspfade analysiert, der Ansatz des Discursive Institutionalism (DI) etabliert, der bei der Analyse der Rolle von Ideen auf die Politikgestaltung und die Neugestaltung institutioneller Arrangements die Ansätze der Diskurstheorie mit denen des Neo-Institutionalismus kombiniert (Schmidt 2008). Die grundlegende Annahme ist, dass institutionelle Dynamiken durch das Auftauchen neuer Ideen, Konzepte und Narrative hervorgerufen werden und die Stabilität institutioneller Arrangements durch das Ausmaß der Institutionalisierung von Ideen, Konzepten und Narrativen erklärt werden kann (Arts/Buizer 2009). Der DI macht hierbei eine analytische Unterscheidung zwischen Diskursen und Institutionen: Neue, kritische Diskurse können existierende bestehende institutionelle Arrangements wandeln oder neu gestalten, andersherum können robuste Institutionen auch das Aufkommen neuer Diskurse und ihren Einfluss auf die Politikgestaltung begrenzen (hier finden sich Rückbezüge auf den Ansatz der Pfadabhängigkeit). Diese Entwicklungen können auf unterschiedlichen Ebenen stattfinden. Beispielsweise haben Diskussionen über die Eignung umweltpolitischer Steuerungsansätze auf europäischer Ebene mit der grundlegenden Neuorientierung im Sinne eines integrierten Managements natürlicher Ressourcen Eingang in die Regularien der WRRL gefunden und damit auch die Bedeutung ökonomischer Ansätze institutionell verankert. Auf nationaler Ebene treffen diese Ideen wiederum auf bestehende Institutionen (d. h. Wasserbehörden) und müssen hier verarbeitet werden.

Ideen, als substanzieller Inhalt von Diskursen, sind auf unterschiedlichen belief-Ebenen (nach Sabatier) angesiedelt. Auf der Ebene von deep core beliefs betreffen sie Weltbilder und Philosophien, auf der Ebene von policy core beliefs Problemwahrnehmungen und grundlegende programmatische Ausrichtungen und auf der Ebene von instrumental beliefs letztlich konkrete politische Instrumente und Lösungsansätze (Schmidt 2008). Eine erste Analyse von Ideen und Interessen im Kontext der ökonomischen Anforderungen der WRRL konnte beispielsweise zeigen, dass insbesondere die grundlegenden Überzeugungen einer verstärkten Nutzung ökonomischer Bewertungen im wasserwirtschaftlichen Verwaltungshandeln entgegenstehen (Dehnhardt 2014). Diskurse wiederum sind interaktive, auch konfliktäre Prozesse zur Vermittlung von Ideen (Schmidt 2008), und werden definiert als ein ,... ensemble of ideas, concepts and categories through which meaning is given to social and physical phenomena, and which is produced and reproduced through an identifiable set of practices" (Hermwille 2016,
S. 238). Für die Diskursanalyse sind vor allem die hier vermittelten Ideen und Weltbilder von Interesse.

Die Feststellung, dass Ideen bestehende Institutionen wandeln (können), beantwortet jedoch noch nicht die Frage, WIE Ideen die Politikgestaltung beeinflussen und unter welchen Bedingungen möglicherweise auch kein politischer Wandel zustande kommt. In einer Sonderausgabe des Journal of European Public Policy (2016, 23/3) wird in diesem Zusammenhang vor allem die Frage der Macht betont. So definieren Carstensen und Schmidt (2016, S. 318) ,... ideational power [...] as the capacity of actors to influence other actors' normative and cognitive beliefs through the use of ideational elements" und unterscheiden dabei die "power through ideas" (als rhetorische Macht), die "power over ideas" (als epistemische Macht) und die „power in ideas" (als institutionelle Macht). Blyth` (2016) Analyse zufolge sind trotz der konzeptionellen Stärke dieses Ansatzes gerade diese unterschiedlichen Dimensionen der Macht analytisch schwer zu fassen und er schlussfolgert, dass die ideelle Macht vor allem durch zwei Faktoren begünstigt wurde: zum einen durch die eine sprachliche Formalisierung der Ideen und zum anderen durch die Etablierung eines professionellen Netzwerks, im Sinne der epistemic communities (nach Haas), womit letztlich die Bedeutung von Akteuren und Netzwerken wieder hervorgehoben wird.

Arts und Buizer (2009) entwickeln auf Basis der Kernelemente des discursive institutionalism den Ansatz der policy arrangements (PA), um die Analyse, wie Ideen, Konzepte und Narrative politische Prozesse beeinflussen, zu operationalisieren. PA sind dabei definiert ,... as the way in which a certain policy domain is temporarily shaped in terms of discourses, actors, resources and rules." (S. 343). Entsprechend werden vier Dimensionen analysiert: Erstens, Policy-Diskurse als Normen, Deutungsmuster und Narrative, die auch konkurrierende Diskurse verschiedener Akteursguppen umfassen können, die sich gegenseitig beeinflussen; zweitens jeweilige Akteure als Diskurskoalitionen sowie drittens formale Prozeduren und informelle Regeln und viertens Macht und Einflussmöglichkeiten verschiedener Akteurskonstellationen, die jeweils nach Hegemonie streben, um ihre präferierte Politik umzusetzen.

\section{Differierende Diskurse in der Umwelt- und Naturschutzpolitik}

Der ÖSL-Ansatz hat sich mittlerweile im akademischen Bereich und der Kommunikation zwischen Wissenschaft und Politik etabliert. Allerdings differieren die Interpretationen über die Bedeutung, die geeigneten Instrumente und das zugrunde liegende Wertkonzept zum Teil erheblich. Jede Disziplin scheint das ESS (ecosystem services) Konzept im Sinne des eigenen Weltbildes zu nutzen und die eigene Position damit zu stärken. Trotz zum Teil sehr emotional geführter Debatten um einen möglichen „Ausverkauf der Natur", sollten ökonomische Instrumente eine stärkere Bedeutung erlangen, verfolgen alle Ansätze und Interpretationen doch das gemeinsame Ziel, 
die Bedeutung des Schutzes der natürlichen Lebensgrundlagen verstärkt auf die politische Agenda zu bringen (Chaudhary et al. 2015, Danley/Widmark 2016). Vor dem Hintergrund der dargestellten Kernelemente des PA-Ansatzes sollen die unterschiedlichen Diskurse um das ÖSL-Konzept und vor allem die Bedeutung ökonomischer Werte sowie deren Wirkung auf die Politikgestaltung skizziert werden.

\section{Diskurse und Diskurskoalitionen}

Der Diskurs um die Interpretation des ÖSL-Konzeptes wird im Wesentlichen von zwei wissenschaftlichen Disziplinen getragen und bestimmt, nämlich der Ökologie und der Ökonomie, die sich in der Folge weiter ausdifferenziert haben (Chaudhary et al. 2015; Spash/Aslaksen 2015; Danley/Widmark 2016). Aus naturwissenschaftlicher - ökologischer - Perspektive zentral ist die Betonung der Kapazität natürlicher Ökosysteme, Funktionen, Prozesse und Komponenten als Grundlage für das menschliche Wohlergehen bereitzustellen. ÖSL werden dabei vollkommen unabhängig von der gesellschaftlichen Nachfrage und vom Nutzen für den Menschen gesehen, vielmehr geht es ausschließlich um die Bereitstellung der natürlichen Lebensgrundlagen und Selbstorganisationsfähigkeit von Ökosystemen, mithin um deren intrinsischen und nicht instrumentellen Wert. Der Fokus liegt entsprechend auf physisch messbaren Indikatoren bei der Darstellung der ÖSL. Auf der anderen Seite steht der Argumentationsrahmen von Umweltökonom/innen. Ausgangspunkt ist hier die Annahme, dass sich Umweltprobleme vor allem aufgrund von Marktversagen erklären lassen, da der Wert öffentlicher, nicht marktlicher Güter nicht bekannt und damit in politischen Entscheidungen bislang unzureichend berücksichtigt wurde. Öffentliche Güter auch als ökonomische Ressourcen zu begreifen und diesen Wert entsprechend zu vermitteln, kann helfen, ÖSL und Biodiversität wieder verstärkt auf die gesellschaftliche und politische Agenda zu bringen. Der ökonomische Wertansatz beruht auf der Ermittlung individueller Präferenzen als Ausdruck der Wertschätzung von ÖSL. Die Bewertung von Wert(bestandteilen) wird dabei als wertvolle Heuristik gesehen, nicht ohne gleichzeitig zu betonen, dass der gesamte Wert der Natur weit mehr umfasst. Die Analyse von Umweltproblemen aus ökonomischer Sicht besitzt eine lange wissenschaftliche und politische Tradition und hat Anfang der 1990er Jahre Eingang in die europäische Umweltpolitik gefunden, durch den Bezug zum ÖSL-Konzept jedoch in den letzten Jahren zusätzliches Gewicht bekommen. Durch das ÖSLKonzept und dessen anthropozentrische Perspektive sind die beiden weitgehend getrennt verlaufenden Diskurse miteinander verknüpft, insbesondere durch den gemeinsamen Bezug auf das menschliche Wohlergehen. Vereinfacht gesagt rückt die ökologisch dominierte Perspektive jedoch vor allem die Angebotsseite, die ökonomische die Nachfrageseite ins Zentrum. Das Feld hat sich in den letzten Jahren um Akteure aus verschiedenen Disziplinen erweitert, die mit der einen oder anderen Seite Koalitionen eingegangen sind. Beispielsweise nimmt die Landschaftsplanung zwar eine anthropozentrische Perspektive ein, die den Nutzen für den Menschen in den Mittelpunkt stellt, leitet diese Ansprüche jedoch aus der Naturschutzgesetzgebung ab und stützt sich bei der Bewertung vor allem auch auf das Dargebot. Die „Umweltpragmatiker“ (Spash/Aslaksen 2015) als relativ neue Akteursgruppe haben die Bedeutung monetärer Werte erkannt („Macht der großen Zahlen“, wie auch die einflussreiche Studie von Costanza 1997) und nutzen diese in einem rein auf instrumentelle Werte ausgerichteten Diskurs, vernachlässigen allerdings eine theoriekonforme Anwendung ökonomischer Methoden und sind daher aus ökonomischer Sicht häufig fragwürdig.

Unabhängig von derartigen Ausdifferenzierungen unterscheiden sich die Diskurskoalitionen nicht nur in ihren grundlegenden Überzeugungen und Problemwahrnehmungen, sondern auch in den präferierten Lösungsansätzen, die gegenwärtig verhindern, dass sich ein weitgehend von allen akzeptiertes ÖSL-Paradigma durchsetzen kann. Entsprechend erweist sich das ÖSL-Konzept gegenwärtig auch (noch) als wenig handlungswirksam.

\section{Institutionen, Regeln und Machtverhältnisse}

Die Bedeutung von ÖSL einerseits und die Ökonomisierung des umweltpolitischen Diskurses andererseits haben Niederschlag in verschiedenen Regularien und Institutionen gefunden. Auf europäischer Ebene zeigt sich der umweltpolitische Einfluss an der Neuausrichtung der Biodiversitätspolitik: vom klassischen Schutzgebietsansatz hin zu einer stärker ökonomisch ausgerichteten Strategie, unter Einbeziehung des Wertes von ÖSL und biologischer Vielfalt und anreizorientierter Steuerungsmechanismen. Die Etablierung der Intergovernmental Platform on Biodiversity and Ecosystem Services (IPBES) im Jahr 2012 stellt einen Meilenstein in der Interpretation und Institutionalisierung des ÖSL-Konzeptes dar, da hier das existierende Wissen zusammengetragen wird und gegenwärtig die wesentlichen Aushandlungsprozesse „... about the allocation of power and authority“ (Russel et al. 2016, S. 586) stattfinden. Die Sozial- und Geisteswissenschaften sind in den IPBES-Expertengruppen und Gremien mit etwa $10 \%$ gegenwärtig jedoch unterrepräsentiert. Im deutschen TEEB-Diskurs erweist sich die Einbindung ökonomischen Expertenwissens als ausgesprochen schwierig. Während in der internationalen TEEB-Studie zu Beginn die Vorstellung stand, in Anlehnung an den SternReport mit Blick auf die Kosten des Klimawandels über die „große Zahl“ wirksam zu werden, und dieser noch stark ökonomisch ausgerichtet war, ist in den nationalen Berichten zum Naturkapital Deutschland eine kontinuierliche Abnahme der Bedeutung umweltökonomischer Ansätze festzustellen. Die begrenzte Wirksamkeit hängt unabhängig von einer begrenzten Akzeptanz ökonomischer Bewertungsverfahren auch damit zusammen, dass das bestehende institutionelle Gefüge das Konzept und die Methoden überhaupt erst aufgreifen und operationalisieren muss. In der Wasserwirtschaft hat sich gezeigt, 
dass die Anforderungen an die Integration ökonomischer Ansätze auf weitgehend stabile politisch-administrative Institutionen stoßen, die weiterhin ihre herkömmlichen Bewertungsformen präferieren und sich den neuen Herausforderungen nur schwer öffnen (Dehnhardt 2013).

\section{Fazit}

Der Schutz der Natur erweist sich auch in der langen Sicht als immer noch schwer umsetzbar. Die Entwicklungsdynamiken gesellschaftlicher Systeme sind offenbar nicht kompatibel mit dem Erhalt und Schutz der Natur und der Ökosysteme. Die Vorstellung, über das Konzept der ÖSL und deren (ökonomische) Bewertung den Schutz der Natur zu ermöglichen, indem der Wert der Natur in die Entscheidungskalküle einfließt und damit die ökonomischen Handlungsrationalitäten im Sinne des Naturschutzes wirksam werden zu lassen, scheinen sich gegenwärtig nur begrenzt durchsetzen zu lassen (auch wenn das United Nations Environment Programme im Kontext der Green Economy die Bewertung von ÖSL als Steuerungsidee vorgeschlagen hat). Die erforderlichen institutionellen Veränderungen, im Sinne einer Einbindung des Konzeptes in bestehende Institutionen oder der Etablierung neuer Institutionen wie IPBES, die dann gegenüber bestehenden Institutionen wirkmächtig werden, ist gegenwärtig eine offene Frage. Insofern trägt die Idee der ÖSL als Brückenkonzept weit und wird vielfach akzeptiert und genutzt, allerdings um den Preis, dass die Konflikte um eine „Ökonomisierung“ der Natur erneut zutage treten und damit letztlich Konflikte um die dahinterliegenden Weltbilder deutlich werden. Eine Ablehnung der ökonomischen Bewertung alleine hilft allerdings keineswegs, die Naturzerstörung aufzuhalten. Insofern ist es notwendig, über konzeptionelle Differenzen hinaus zu gehen. Dieser fragmentarische Blick auf die Rolle von Ideen legt die Vermutung nahe, dass fundamentale Fragen, wie ein unterschiedliches Verständnis von Wissen und dessen politischer Nutzung, neben operationalen Fragen die Übernahme in den politischen Entscheidungsprozess gegenwärtig begrenzen. Vor allem wird aber auch deutlich, dass es noch einen großen Forschungsbedarf dahingehend gibt, wie, von wem und ich welchen Kontexten neues Wissen und ein neues Verständnis vom Wert der Natur in die Politik aufgenommen werden kann.

\section{Literatur}

Arts, B./Buizer, M. (2009): Forests, discourses, institutions. In: Forest Policy and Economics 11/5-6: 340-347.

Béland, D./Carstensen, M. B./Seabrooke, L. (2015): Ideas, Political Power and Public Policy. In: Journal of European Public Policy 23/3: 315-317.

Béland, D./Waddan, A. (2011): Ideen und sozialpolitischer Wandel. Konzeptionelle Überlegungen am Beispiel der USA. In: Zeitschrift für Sozialreform 57/4: 463-485.

Blyth, M. (2016): The New Ideas Scholarship in the Mirror of Historical Institutionalism: A Case of Old Whines in New Bottles? In: Journal of European Public Policy 23/3: 464-471.
Campbell, J. L. (2002): Ideas, Politics, and Public Policy. In: Annual Review of Sociology 28/1: 21-38.

Carstensen, M. B./Schmidt, V. A. (2016): Power through, over and in ideas: conceptualizing ideational power in discursive institutionalism. In: Journal of European Public Policy 23/3: 318-337.

Chaudhary, S./McGregor, A./Houston, D./Chettri, N. (2015): The evolution of ecosystem services. A time series and discourse-centered analysis. In: Environmental Science \& Policy 54: 25-34.

Danley, B./Widmark, C. (2016): Evaluating conceptual definitions of ecosystem services and their implications. In: Ecological Economics 126: 132-138.

Dehnhardt, A. (2012): Die Anwendung von Kosten-Nutzen-Analysen im wasserwirtschaftlichen Verwaltungshandeln - eine empirische Untersuchung im Kontext der EG-Wasserrahmenrichtlinie. In: Zeitschrift für Umweltpolitik und Umweltrecht (ZfU) 3/2012: 283-311.

Dehnhardt, A. (2013): Decision-makers' Attitudes towards Economic Valuation - a Case Study of German Water Management Authorities. In: Journal of Environmental Economics and Policy 2/2: 201-221.

Dehnhardt, A. (2014): The Influence of Interests and Beliefs on the Use of Environmental Cost-Benefit Analysis in Water Policy. The case of German policy-makers. In: Environmental Policy and Governance 24/6: 391-404.

Hermwille, L. (2016): The role of narratives in socio-technical transitions Fukushima and the energy regimes of Japan, Germany, and the United Kingdom. In: Energy Research \& Social Science 11: 237-246.

Laurans, Y./Mermet, L. (2014): Ecosystem services economic valuation, decision-support system or advocacy? In: Ecosystem Services 7: 98-105.

MA (2005): Millennium Ecosystem Assessment - Ecosystems and human well-being. Synthesis. Washington, D. C.: Island Press (Millennium Ecosystem Assessment Series).

Martin-Ortega, J. (2012): Economic prescriptions and policy applications in the implementation of the European Water Framework Directive. In: Environmental Science \& Policy 24: 83-91.

Naturkapital Deutschland - TEEB DE (2012): Der Wert der Natur für Wirtschaft und Gesellschaft - eine Einführung. München, ifuplan; Leipzig, Helmholtz-Zentrum für Umweltforschung - UFZ; Bonn, Bundesamt für Naturschutz.

Russel, D./Jordan, A./Turnpenny, J. (2016): The Use of Ecosystem Services Knowledge in Policy-Making. In: Potschin, M./Haines-Young, R./Fish, R./ Turner, R. K. (Hrsg.): Routledge Handbook of Ecosystem Services. London, Routledge: 586-596.

Schmidt, V. A. (2008): Discursive Institutionalism. The Explanatory Power of Ideas and Discourse. In: Annual Review of Political Science 11/1: 303-326.

Spash, C. L./Aslaksen, I. (2015): Re-establishing an ecological discourse in the policy debate over how to value ecosystems and biodiversity. In: Journal of environmental management 159: 245-253.

\section{AUTOREN + KONTAKT}

Dr. Alexandra Dehnhardt ist Mitarbeiterin am Fachgebiet Landschaftsökonomie des Instituts für Landschaftsarchitektur und Umweltplanung der Technischen Universität Berlin.

TU Berlin, Fachgebiet Landschaftsökonomie, Straße des 17. Juni 145, 10623 Berlin. Tel.: +49 30 314-21358, E-Mail: alexandra.dehnhardt@tu-berlin.de

Ulrich Petschow ist Leiter des Forschungsfeldes Umweltökonomie und Umweltpolitik am Institut für ökologische Wirtschaftsforschung (IÖW).

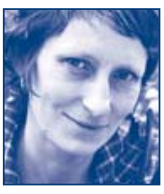

IÖW, Potsdamer Str. 105, 10785 Berlin.

Tel.: +49 30 884594-0, E-Mail: ulrich.petschow@ioew.de, Internet: www.ioew.de 